\title{
Dynamic modulation of local population activity by rhythm phase in human occipital cortex during a visual search task
}

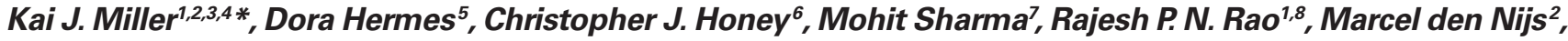 Eberhard E. Fetz ${ }^{1,9}$, Terrence J. Sejnowski ${ }^{10}$, Adam O. Hebb ${ }^{3}$, Jeffrey G. Ojemann ${ }^{3}$, Scott Makeig ${ }^{11}$ and Eric C. Leuthardt ${ }^{7,12}$}

\author{
1 Neurobiology and Behavior, University of Washington, Seattle, WA, USA \\ 2 Physics, University of Washington, Seattle, WA, USA \\ 3 Neurological Surgery, University of Washington, Seattle, WA, USA \\ 4 Johnson Space Center, Houston, TX, USA \\ ${ }_{5}$ Rudolf Magnus Institute of Neuroscience, University Medical Center Utrecht, Utrecht, Netherlands \\ ${ }^{6}$ Department of Psychology and Princeton Neuroscience Institute, Princeton University, Princeton, NJ, USA \\ 7 Biomedical Engineering, Washington University, St. Louis, MO, USA \\ ${ }^{8}$ Computer Science and Engineering, University of Washington, Seattle, WA, USA \\ ${ }_{9}$ Physiology and Biophysics, University of Washington, Seattle, WA, USA \\ ${ }^{10}$ Howard Hughes Medical Institute, The Salk Institute, San Diego, CA, USA \\ ${ }^{11}$ Division of Biological Sciences, University of California, San Diego, CA, USA \\ ${ }^{12}$ Neurological Surgery, Washington University, St. Louis, MO, USA
}

\section{Edited by:}

Thilo Womelsdorf, Robarts Research

Institute London, Canada

Reviewed by:

Tobias H. Donner, University of

Amsterdam, Netherlands

Michael Breakspear, Queensland

Institute of Medical Research, Australia

*Correspondence:

Kai J. Miller, Department of Physics, University of Washington, Box 351560 ,

Seattle, WA 98195, USA.

e-mail:kjmiller@u.washington.edu; kjmiller@gmail.com
Brain rhythms are more than just passive phenomena in visual cortex. For the first time, we show that the physiology underlying brain rhythms actively suppresses and releases cortical areas on a second-to-second basis during visual processing. Furthermore, their influence is specific at the scale of individual gyri. We quantified the interaction between broadband spectral change and brain rhythms on a second-to-second basis in electrocorticographic (ECoG) measurement of brain surface potentials in five human subjects during a visual search task. Comparison of visual search epochs with a blank screen baseline revealed changes in the raw potential, the amplitude of rhythmic activity, and in the decoupled broadband spectral amplitude. We present new methods to characterize the intensity and preferred phase of coupling between broadband power and band-limited rhythms, and to estimate the magnitude of rhythm-to-broadband modulation on a trial-by-trial basis. These tools revealed numerous coupling motifs between the phase of low-frequency $(\delta, \theta, \alpha, \beta$, and $\gamma$ band) rhythms and the amplitude of broadband spectral change. In the $\theta$ and $\beta$ ranges, the coupling of phase to broadband change is dynamic during visual processing, decreasing in some occipital areas and increasing in others, in a gyrally specific pattern. Finally, we demonstrate that the rhythms interact with one another across frequency ranges, and across cortical sites.

Keywords: electrocorticography, occipital cortex, vision, broadband, rhythm, phase-amplitude coupling, nested oscillation, beta

\section{INTRODUCTION}

Recent electrocorticographic (ECoG) and local field potential (LFP) measurements have revealed a link between neuronal population activity and changes in broadband spectral power in the brain surface electric potential (Manning et al., 2009; Miller et al., 2009b; Whittingstall and Logothetis, 2009). This broadband change has been studied primarily by examining power at higher frequencies, where underlying brain rhythms $(\delta, \theta, \alpha, \beta$, and low- $\gamma)$ do not obscure behaviorally-modulated broadband changes (Brindley and Craggs, 1972; Crone et al., 2001; Edwards et al., 2009; Miller et al., 2009a, 2010a; Jacobs and Kahana, 2010), and more recently by decomposition of spectral changes to remove the rhythms (Miller et al., 2009c). These brain rhythms have long been known to fluctuate with behavior (Beck, 1891), but whether they reflect an active or passive agent in cortical computation has not been directly addressed until recently. Recent measurements have demonstrated that the phase of low-frequency rhythms modulates the amplitude of high frequency power in ECoG (Mormann et al., 2005; Canolty et al., 2006; Osipova et al., 2008; Penny et al., 2008; He et al., 2010). This finding implies that the physiological mechanisms underlying a particular brain rhythm are influencing neuronal population activity recorded at the cortical surface, and further investigation may therefore yield insight into large-scale information processing motifs in the brain. These previous studies have focused on phase modulation of broadband amplitudes on the timescale of minutes. The examination of modulation at finer temporal resolution may reveal how information is dynamically processed in the brain on the timescale at which neural circuits engage and disengage with transient stimuli.

With a single exception (He et al., 2010), previous reports of these types of nested oscillations have focused on coupling between the phase of a low-frequency rhythm and a "high-gamma" range (Mormann et al., 2005; Canolty et al., 2006; Osipova et al., 2008; Penny et al., 2008; Cohen et al., 2009). Close inspection of the figures 
in these manuscripts indicates that this "high-gamma" may be a reflection of broadband change, and not a band-limited $\gamma$-rhythm. It is important to draw a distinction between broad band activity and higher frequency $\gamma$-rhythm $(>50 \mathrm{~Hz})$ amplitude changes as they may reflect distinct underlying physiologic phenomena. The $\gamma$-rhythm has been suggested to play a role in active computation in the brain by synchronization - associating distinct cortical sites coherently to facilitate computation (Singer, 1993; Fries et al., 2001; Womelsdorf et al., 2005, 2007; Siegel et al., 2008; Mitchell et al., 2009). In contrast to playing a specific computational role, the broadband power measured at an electrode reflects the average spike rate of the neurons that project to the local neuronal population of roughly $5^{\star} 10^{5}$ neurons in the gray matter beneath each electrode (Manning et al., 2009; Miller et al., 2009b; Miller, 2010). Any phenomena that are observed in the aggregate potential therefore reflect the general activity of this underlying population.

Universal functional properties are often attributed to bandlimited rhythmic phenomena. In some cases, this might be biologically plausible - the $\theta$ rhythm, for example, may enable a central hippocampal mechanism to coordinate a distributed neocortical network during planning and memory retrieval (Mormann et al., 2005; Canolty et al., 2006; Tort et al., 2009). The $\alpha$ rhythm may reflect suppressive synchronization across visual cortex when there is no visual input at all (Osipova et al., 2008), and therefore not a major feature of our task (as described below), where the subjects' eyes were open throughout both visual search and ISI epochs. Emerging experimental results suggest that the $\alpha$-rhythm may not emerge as a large-scale brain feature in visual cortex until the signal is averaged over the larger spatial scales associated with EEG and MEG recordings (Edwards, 2010, Personal Communication).

In this article, we lay out a general framework for examining modulation of local population activity by low-frequency rhythms in the brain. A "phase-coupling palette" is constructed to visualize the full range of coupling across a wide range of frequencies. We isolate particular frequency ranges, and demonstrate how trial-bytrial modulation can be quantified in a robust way using the "phasecoupling vector." These measures reveal numerous coupling motifs between low-frequency rhythms and broadband spectral change in human occipital cortex. This coupling changes dramatically on a trial-by-trial basis as individuals switch between engagement in a visual search task and fixation on a blank screen. Our findings suggest that rhythms have multiple influences, facilitating and suppressing the activity of cortical populations of neurons. Within a specific frequency range, these influences can be gyrally specific, releasing one area during visual engagement, while actively suppressing another.

\section{METHODS \\ SUBJECTS}

All five subjects in the study were epileptic patients at Harborview Hospital in Seattle, WA. Sub-dural grids and strips of platinum electrodes were clinically placed over frontal, parietal, temporal, and occipital cortex for extended clinical monitoring and localization of seizure foci. Each subject gave informed consent to participate in an Institutional Review Board approved experimental protocol. All patient data was anonymized according to IRB protocol, in accordance with HIPAA mandate.

\section{RECORDINGS}

Experiments were performed at the bedside, using Synamps2 amplifiers (Neuroscan, El Paso, TX, USA) in parallel with clinical recording. Stimuli were presented with a monitor at the bedside using the general purpose BCI2000 stimulus and acquisition program (interacting with proprietary Neuroscan software), which also recorded the behavioral parameters and cortical data. Subdural platinum electrode arrays (Ad-Tech, Racine, WI, USA) were arranged as combinations of $8 \times[4,6,8]$ rectangular fronto-tempero-parietal arrays and $1 \times[4,6,8]$ linear temporal and occipital strips. The electrodes had $4 \mathrm{~mm}$ diameter $(2.3 \mathrm{~mm}$ exposed $), 1 \mathrm{~cm}$ inter-electrode distance, and were embedded in silastic. The potentials were sampled at $1000 \mathrm{~Hz}$, with respect to a scalp reference and ground (Figure 1A). These signals had an instrument-imposed band-pass filter from 0.15 to $200 \mathrm{~Hz}$.

\section{VISUAL SEARCH TASK}

Subjects participated in a visual search task presented on a LCD monitor $\sim 1 \mathrm{~m}$ away, in which $2 \mathrm{~s}$ visual search stimuli were interleaved with $2 \mathrm{~s}$ inter-stimulus-intervals (ISIs) during which the screen was blank. Each visual search stimulus (e.g., Figure 1B) consisted of three parts: (1) a 5-row by 4-column array of colored boxes $(\sim 1 \mathrm{~cm}$ by $1 \mathrm{~cm}),(2)$ a white star positioned in the center of one of these boxes, and ( 3 ) a black arrow $(-2 \mathrm{~cm}$ by $1 \mathrm{~cm})$ centered $\sim 1.5 \mathrm{~cm}$ to the right of the right-most box in the middle row. In each visual search stimulus, the star appeared randomly in one of the colored boxes, and the arrow pointed randomly in one of four cardinal directions ("right," "left," "up," or "down"). The subjects' task was to state the color of the box that was adjacent to the star in the direction of the arrow. Since fixation was not constrained, and eye position was not measured, we cannot know whether differential effects between different arrow directions were due to attention or a late visual microsaccade between the star and the arrow, or different box position. It seems unlikely that we are measuring "motor processing" in visual cortex during visual search, but we cannot exclude this possibility. Because the uncertainty about attention versus gaze-shift-driven neural activity limits our ability to examine the functional subcomponent of visual processing, the present study is agnostic to these distinctions, and is focused on the generic relationship between brain rhythms and cortical visual processing.

\section{ELECTRODE LOCALIZATION}

Cortical surface mesh reconstructions were made using preoperative structural MRI. Electrode positions were calculated with respect to the structural MRI from post-operative computed tomography (CT) using the CTMR package of Hermes et al. (2010). When the MRI or CT was of insufficient quality, hybrid techniques involving $\mathrm{X}$-ray were employed to obtain cortical rendering and/or electrode position (Miller et al., 2010b).

\section{SIGNAL PROCESSING}

Complex signals $\tilde{V}(f, t)=r(f, t) \mathrm{e}^{i \phi(f, t)}$ at discrete frequencies, $f$, were extracted from the raw potential using Morlet wavelets, and complex signals representing a range of frequencies $\tilde{V}(F, t)=r(F, t) \mathrm{e}^{i \phi(F, t)}$ were generated using a band-pass and then the Hilbert transform. The log of the decoupled broadband amplitude, $\chi(t)$, was also 
extracted from the raw potential, using the approach outlined in Miller et al. (2009c). The methods for pre-processing, and other pre-established signal processing, are detailed in the Methodological Appendix. Synthetic data were generated to validate the signal processing approach, and the methods to do this are also detailed in the Appendix.

\section{BROADBAND COUPLING TO LOW-FREQUENCY PHASE (ILLUSTRATED IN FIGURE 1D-F)}

The coupling was estimated by calculating the average log-broadband amplitude $\chi(t)$ as a function of the rhythm phase $\phi$ in small phase intervals,

$\chi_{k}=\langle\chi(t)\rangle_{\left(k^{\prime}+1 / 2\right)>\frac{\phi(t)}{\pi} \geq\left(k^{\prime}-1 / 2\right)}$,

where $k^{\prime}=k-K / 2$ ( $K$ total intervals). For example, for $K=24$ (the number used in these analyses), then $\chi_{13}$ represents the mean log-broadband when the phase of the low-frequency rhythm is in the interval between 0 and $\pi / 12$. The center of each interval is denoted $\phi_{k}$, so $\phi_{13}=\pi / 24$. To get a full picture of the strength and preferred phase of coupling across a range of frequencies, the wavelet-obtained rhythms at each frequency are used to build up a "palette" of $\chi_{k}$ at each frequency. This has an advantage in that ranges of coupling and distinct coupling to different rhythms are revealed, in many cases, as separate phenomena, because there are different preferred phases of coupling.

\section{THE COUPLING VECTOR AND TRIAL-BY-TRIAL STATISTICS (ILLUSTRATED IN FIGURE 2)}

To condense the range of frequencies composing a given rhythm into one measure, the Hilbert transform was applied, with frequency range chosen based upon inspection of the palette. This allows for the calculation of a "coupling vector" by taking the dot-product $Z_{\text {mod }} \mathrm{e}^{i \phi_{c}}=1 / 2 K \sum_{k} \chi_{k} \mathrm{e}^{i \phi_{k}}$. $Z_{\text {mod }}$ is the magnitude of coupling between phase of the rhythm and the log-broadband amplitude (because we $z$-score $\chi(t), Z_{\text {mod }}$ is roughly the amount of variation in the $z$-score that correlates with the phase of the rhythm concerned), and $\phi_{c}$ is the preferred phase of this interaction. This can be calculated on a trial-by-trial basis, breaking up the data into smaller epochs ( $2 \mathrm{~s}$ trials in our case) and calculating a coupling vector for each trial. To assess the distribution of coupling for $N$ trials of a given type, one cannot simply compare the contribution of trial $n$ to the distribution of coupling values as $Z_{\bmod }(n)$ because if $\phi_{c}$ is not reproducible from trial-to-trial, then $\overline{Z_{\text {mod }}(n)}$ can be a large value even on trials in which the coupling preferred phase is opposite to that of the majority of other trials in the distribution. In other words, the fact that $\overline{Z_{\bmod }(n)}$ must be non-negative would strongly bias the distribution of $\overline{Z_{\text {mod }}(n)}$ values so that the mean of the distribution can be significantly greater than zero even when there would be no underlying coupling of consistent phase. For this reason, the distribution of values $Z_{\bmod }^{q}(n)=Z_{\bmod }(n) * \cos \left(\phi(n)-\overline{\phi^{q}}\right)$ is used, where $\overline{\phi^{q}}$ is the preferred phase of the mean coupling vector for trials of type $q: \overline{Z_{\text {mod }}^{q}} \mathrm{e}^{i \bar{\phi}^{q}}=1 / N \sum_{n} Z_{\text {mod }}(n) \mathrm{e}^{i \phi(n)}$ The quantity $Z_{\text {mod }}^{q}$ can be negative or positive and can therefore have a distribution significantly overlapping with zero (indicating an absence of reliable phase modulation). For each type of trial, the distribution of coupling can be assessed using the distribution $Z_{\text {mod }}^{q}(n)$. We demonstrate the significance of these measures using error bars which represent three times the standard error of the mean. Note that if all trials are of type $q$, then the average coupling vector across trials is the same as the coupling vector of the full timeseries. This method has an advantage over the "large-time shift" bootstrapping approach (Penny et al., 2008) because the data may be segregated into smaller time trials, and statistics may be computed by comparing the distribution of values for trials of one behavioral state versus trials of a different behavioral state. It may also be used to compare discontinuous trials of one type (or all trials concatenated) versus 0 . This allows us to examine significant shifts in phaseamplitude coupling during different trial types (where $q=$ "ISI," versus $q$ = "right," etc.) and also to assess the significance of each independently versus zero. One might in principle look for shifts in the distribution of preferred phase, but this is only meaningful in the case that significant coupling versus zero has been established independently for each of the two distributions. We defer examination of task-related shift in preferred phase to future studies.

\section{CROSS-RHYTHM INTERACTION}

We also calculated the interaction between the 4-8 Hilbert phase $\phi(4-8 \mathrm{~Hz}, t)$, and the $12-20 \mathrm{~Hz}$ analytic amplitude $r(12-20 \mathrm{~Hz}, t)$. This was done in the exact same fashion as for the $\phi$ to $\chi$ comparison, replacing $\chi$ with $r(12-20 \mathrm{~Hz}, t)$. There are many such interrhythm interactions (e.g., Lakatos et al., 2005; Osipova et al., 2008; Cohen et al., 2009; Tort et al., 2009), but we defer further explorations of these to future studies.

\section{RESULTS}

TASK-SPECIFIC CHANGES IN THE RAW POTENTIAL, THE AMPLITUDE OF RHYTHMS, AND IN DECOUPLED LOG-BROADBAND SPECTRAL CHANGE BETWEEN VISUAL SEARCH AND INTER-STIMULUS INTERVAL EPOCHS Averaged power spectra

The averaged power spectral density (PSD) during visual engagement and ISI exhibits a general $1 / f$ shape, with superimposed rhythms that deviate from this $1 / f$ structure at particular frequencies. During behavior, these averaged PSDs most commonly reveal decreases in power in the classic $\theta(\sim 4-8 \mathrm{~Hz}), \alpha(\sim 8-12 \mathrm{~Hz})$, and $\beta(\sim 12-20 \mathrm{~Hz})$ rhythms during visual engagement, and increases in power in the $\gamma$ rhythm $(30-50 \mathrm{~Hz})$, and in broadband change (Figures $1 \mathrm{C}$ and $4 \mathrm{H}, \mathrm{I}$ ).

\section{Dynamic power spectra}

The dynamic spectra shown in Figures 4 and 5 reveal that there are both broadband spectral changes, and corresponding increases and decreases in the $\theta / \alpha / \beta / \gamma$ ranges associated with the onset of visual search stimuli.

\section{Decoupling the cortical power spectrum}

A principal component decomposition of the PSD clustered rhythmic, spectrally peaked, phenomena in the 2nd to 4th principal components (Figure 1C). Reconstruction of the PSD without these components revealed broadband spectral change, across the entire frequency domain, with an approximately $1 / f$ structure. This broadband phenomenon is distinct from a higher $\gamma$-rhythm occurring as a peaked activity at $50 \mathrm{~Hz}$ and above (see Figures $4 \mathrm{H}, \mathrm{I}$ ). 
A

A
B

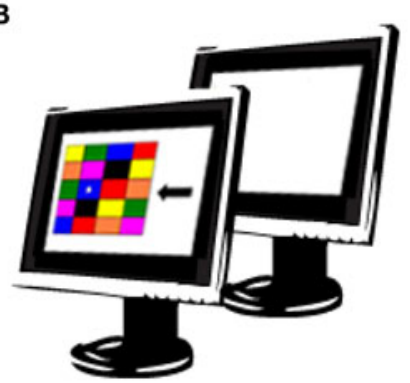

C

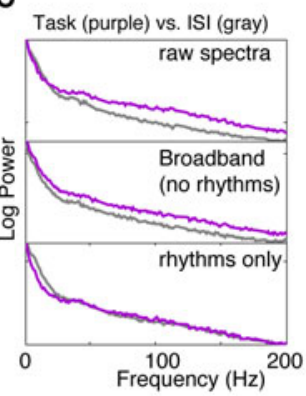

Cues

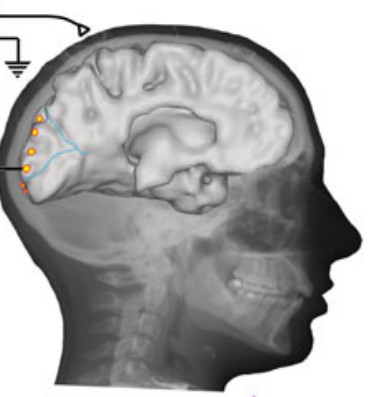

Broadband

Broadband

$\lambda \sqrt{ }$

西

intm

$5 \mathrm{~Hz}$ signal (colored by phase)

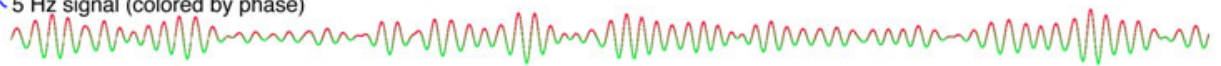

Log Broadband (colored by $5 \mathrm{~Hz}$ phase)

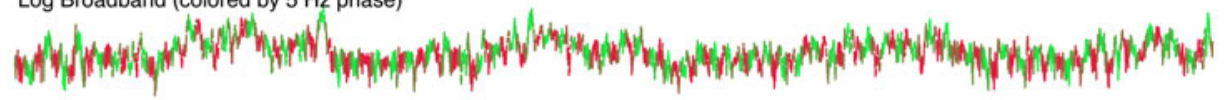

0

2

6

$8 \quad 10$

Time (s)

E

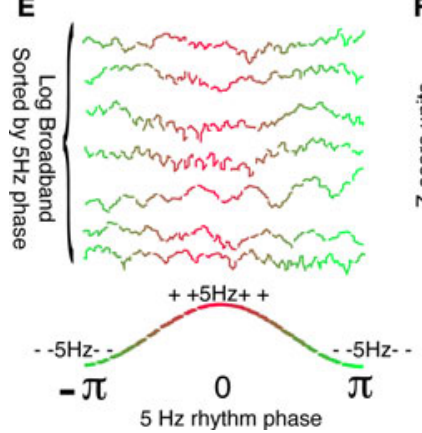

$\mathbf{F}$

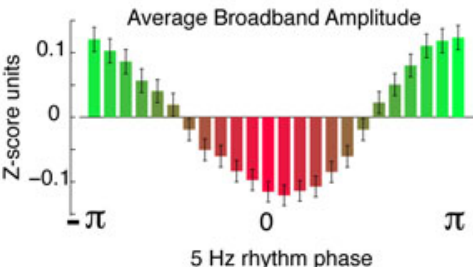

$5 \mathrm{~Hz}$ "coupling row"
G

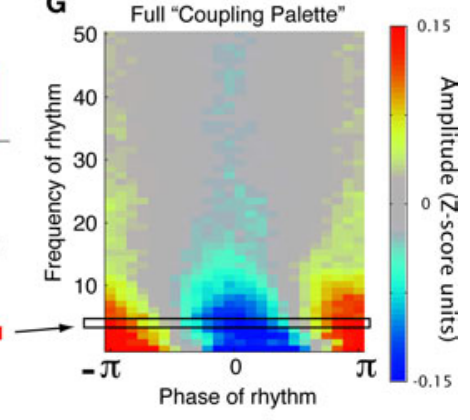

FIGURE 1 | Experimental approach. (A) The electrocorticographic (ECOG) potential is measured from occipital lobe brain surface sites during participation in a visual search task (subject 4 shown here). (B) The visual search task consists of a 4-by-5 array of colored squares. Each cue consists of a star in one of the boxes (in this case a blue box), and an arrow. The subject states the color of the box in the direction of the arrow from the star ("green" would be the appropriate response in this case). Visual search cues are shown for $2 \mathrm{~s}$, with blank inter-stimulus-interval (ISI) cues shown for intervening $2 \mathrm{~s}$. (C) Power spectral changes during different portions of the experiment (top panel) may be appreciated and naively decoupled into broadband changes (middle panel, 2nd to 4th PSCs omitted) and changes in brain rhythms (bottom panel, 2nd to 4th PSCs only). (D) From the timeseries of the potential, dynamic, spectrally-broadband, fluctuations may be extracted (here shown smoothed). In this illustration, the $5 \mathrm{~Hz}$ portion of the signal is extracted using a $5 \mathrm{~Hz}$ Morlet wavelet (here colored for phase - red denotes surface-positive, and green denotes surface-negative). The timeseries of the log of the broadband is shown, colored by coincident phase of the $5 \mathrm{~Hz}$ signal.

(E) The timeseries of the log of the broadband is sorted by the coincident phase of the $5 \mathrm{~Hz}$ signal. (F) The average of the log-broadband amplitude ( $Z$-score units, denoted $\chi_{k}$ in the Methods) for each $5 \mathrm{~Hz}$ phase is shown, with errorbars denoting three times the standard error of the mean ( $3 * \mathrm{SEM})$ for each phase. This can be appreciated in one dimension as the " $5 \mathrm{~Hz}$ coupling row." (G) The process detailed in (D)-(F) is repeated at each frequency from 1 to $50 \mathrm{~Hz}$, to obtain a full coupling palette, and to illustrate how the full range of low-frequency rhythms modulates local brain activity (as captured by broadband spectral change).

\section{Extraction of time-varying broadband change}

A time-varying estimate of broadband spectral change was obtained by projecting the dynamic spectrum into the first principal spectral component (Figure 1D). The broadband change approximates the time-varying amplitude function $A(t)$ in the power-law relationship: $P(t) \sim A(t)\left(1 / f^{x}\right)$. In early visual areas of the occipital cortex (peri-calcarine and occipital pole), this phenomenon is augmented throughout the entire visual search task, and most strongly so at cue onset (Figures 4 and 5). In other areas, such as portions of the lingual gyrus, this phenomenon is suppressed (Figure 5H). This broadband measure is a robust correlate of local cortical activity (Manning et al., 2009; Miller et al., 2009b,c; Miller, 2010) and its modulation by the phase of low-frequency rhythms is the focus of this paper. 


\section{Event-related potentials}

The event-related potentials reveal characteristic voltage deflections at the onset and offset of visual stimuli. These were transient and non-specific, and present whether the corresponding broadband signal increased or decreased (Figures 4 and 5).

\section{Trial-by-trial amplitude measures}

The average and $3{ }^{*}$ SEM amplitudes of epoch-averaged broadband change reveal both increases and decreases (Figures 4 and 5) during visual search periods when compared with ISI epochs. Early visual areas show characteristic increases in power, while other areas show decrease. As shown in Figure 4, these trends cluster by gyral location. Amplitudes of the 12-20 Hz amplitude (Figures 4 and 5) also reveal both taskrelated increases and decreases in amplitude during visual search engagement, but these are not linked to broadband change in any simple way. There is task-related increase in the 4-8 $\mathrm{Hz}$ amplitude (Figure 5) - although this phenomenon was not universal across subjects nor brain area.

\section{THERE ARE NUMEROUS COUPLING MOTIFS BETWEEN LOW-FREQUENCY RHYTHMS AND BROADBAND SPECTRAL CHANGE}

Figure 1 demonstrates that robust modulation of broadband power can be identified across a range of different rhythms by visualizing palettes of rhythm-to-broadband coupling. These palettes display the range of frequencies that are coupled, and the predominant phase of this coupling. Figure 3 shows a strong and significant modulation of broadband spectral change with the phase of lowfrequency rhythms in the $\delta / \theta / \alpha / \beta / \gamma$ ranges, and the relative magnitude of each change at different sites in occipital cortex. These modulatory rhythms often superimpose at the same cortical sites, and can often be appreciated as separate phenomena by the different preferred phase of coupling.

- $\delta(\sim 1-3 \mathrm{~Hz})$ most often has a preferred coupling phase of $-\pi / 4$ to $-\pi / 2$, although there are clear exceptions in subjects 4 and 5 (left-most lower palette in Figures 3 and 4D) where the preferred phase is $\pi$.

- $\quad \theta(\sim 4-8 \mathrm{~Hz})$ has a preferred coupling phase of $\pi$ which appears robustly across subjects and electrodes. (Although Figure 4G is a notable exception in this and other rhythm cases).

- $\quad \alpha(\sim 8-12 \mathrm{~Hz})$ most often has a preferred coupling phase of $\pi / 2$ to $3 \pi / 4$, although this is not universal.

- $\quad \beta(\sim 12-20 \mathrm{~Hz}$, "low- $\beta$ " although particular range appears variable between sites) most often has a preferred coupling phase of $3 \pi / 4$. There are notable exceptions to this (Figure 3 - row 4 , column 2; Figure 4G).

- "Canonical $\gamma$ " (30-50 Hz) coupling is observed in Figures 2-4 with various preferred phases.

It is possible that, in some cases, the smooth, continuous, "diagonal bands" visible in the lower frequencies of the phase-coupling palettes $(\delta, \theta, \alpha$ bands) may not reflect a set of distinct couplings between band-limited low-frequency rhythms and broadband power. Instead, they may reflect a process in which broadband power is elevated at a fixed time lag relative to the peak of the voltage trace, and this fixed time lag then appears as a continuously varying phase lag across different low-frequency bands.
THE AMPLITUDES AND PHASES OF RHYTHMS IN ADJACENT CORTICAL SITES ARE CORRELATED WITH ONE ANOTHER

As shown in Figure 4J, the phase coherence in the $12-20 \mathrm{~Hz}$ range is strong and significant in adjacent sites throughout the 7-electrode strip for subject 5 (note that this is not simply a result of re-referencing, because 63 electrode sites contributed to the common average, not just the 7 shown). This coherence is significant throughout, but is less during visual search task - which might partially be a product of the lower rhythm amplitude in a noise-like background. The coherence is sustained better across neighboring electrodes on the same gyrus. The correlation in the time course of the amplitude of the rhythms in adjacent electrodes is also significant (Figure $4 \mathbf{K}$ ), and is significantly increased during visual search engagement in only three co-gyral electrodes (Figures 4B-D).

\section{THE BROADBAND AMPLITUDE IS SIGNIFICANTLY MODULATED BY THE PHASE OF LOW-FREOUENCY RHYTHMS}

When the potential time series is separated into behaviorally relevant epochs (as described in Figure 3), there is significant modulation during both visual search cues and inter-stimulus intervals. This is shown explicitly for a $4-8 \mathrm{~Hz}$ band and a $12-20 \mathrm{~Hz}$ band (Figures 4 and 5).

\section{THE MODULATION OF BROADBAND POWER BY RHYTHM PHASE SHOWS TASK-DEPENDENT INCREASES AND DECREASES}

The 12-20 Hz range simultaneously shows both increases and decreases in modulation with engagement in the visual search task, and these patterns cluster gyrally (Figures 4 and 5). The visual search induced modulation decreases are most often associated with "active" brain areas, where broadband power increases during visual search.

\section{THE PHASE OF ONE RHYTHM CAN ALSO MODULATE THE AMPLITUDE OF ANOTHER, IN A TASK-DEPENDENT MANNER}

In each subject, modulation of the $12-20 \mathrm{~Hz}$ amplitude by the 4-8 Hz phase was observed, and changed during visual search in some cases (Figures 5 and 6). Such inter-rhythm modulation appeared in at least one site in every subject, but was not present in most sites.

\section{SIMULATED DATA SUPPORTS SIGNAL PROCESSING APPROACH}

A heuristic model was used to create synthetic data with broadband $1 /$ f properties and modulation by a rhythmic input (Figures 7 and 8). This synthetic data was naively separated by the data analysis methods used on cortical data. The resulting spectra, palette, and modulation measures clearly resemble those found from recorded brain signals. When a negative control was analyzed (Figure 9), there is a complete lack of coupling structure.

\section{DISCUSSION}

Our most significant and novel finding is that local cortical activity is dynamically modulated by the phase of different brain rhythms, tracking cognitive engagement on a second-to-second basis for specific cortical sites. In the occipital cortex this modulation was most pronounced in the $\theta(4-8 \mathrm{~Hz})$ and $\beta(12-20 \mathrm{~Hz})$ ranges during periods of relative cognitive disengagement and dissolves during engagement in a visual search task. This relative increase 
A
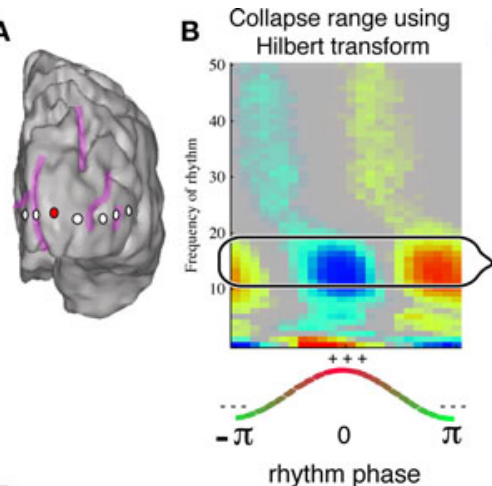

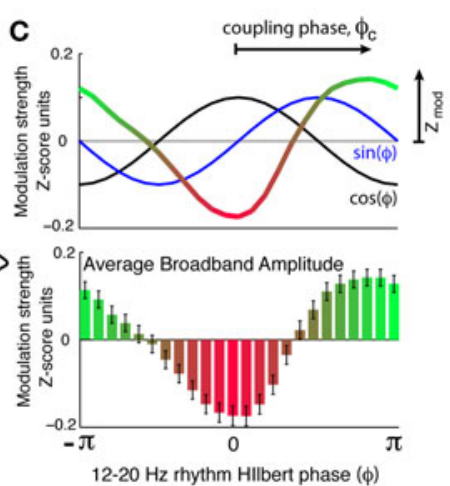

D

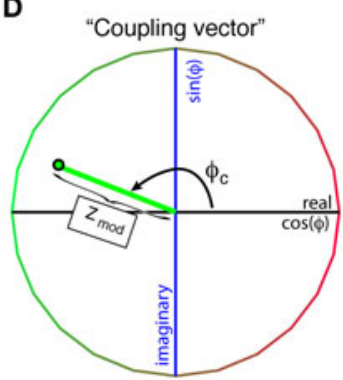

E

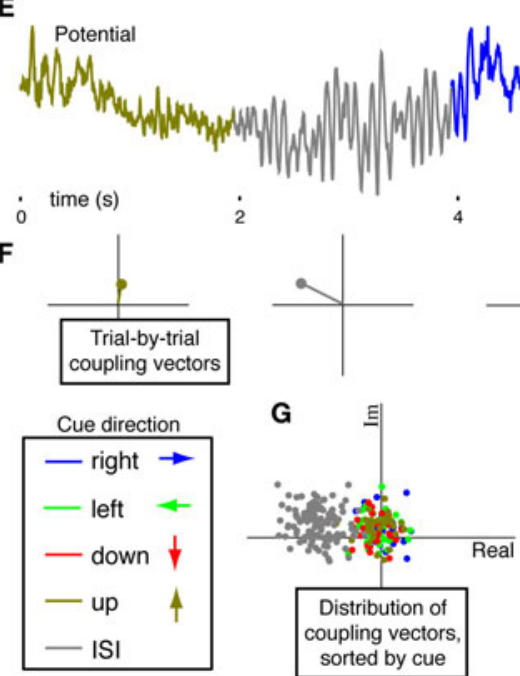

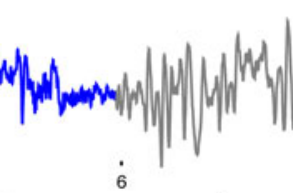

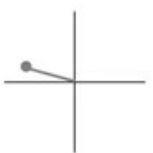

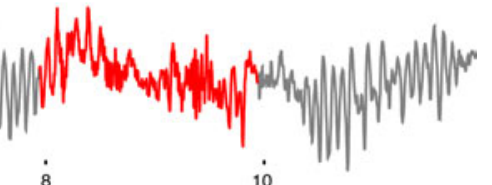

$\dot{8}$

io
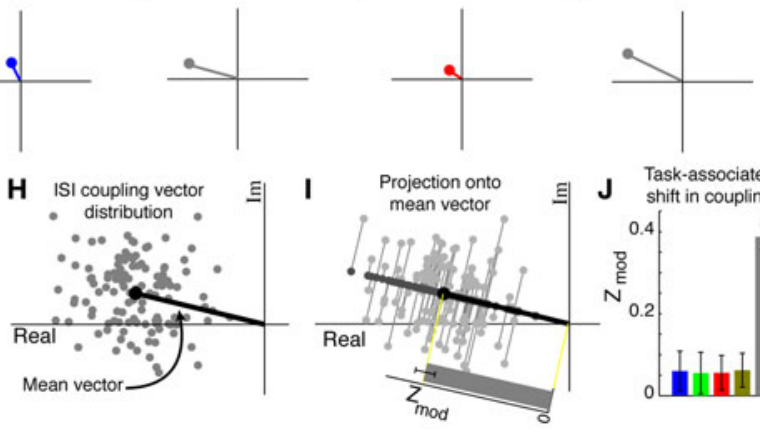

Task-associated

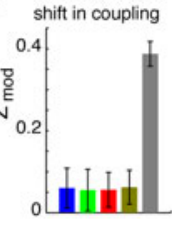

FIGURE 2 |The "coupling vector" and trial-by-trial characterization of modulation. (A) A selected electrode (red) on the subject 5 cortical surface. (B) The coupling palette for this electrode. Phase-amplitude coupling across a range of low- $\beta$ band frequencies $(12-20 \mathrm{~Hz})$, rather than at a single frequency, may be captured using the Hilbert transform rather than Morlet wavelets. (C) Coupling of broadband power to the Hilbert phase of the band-passed $12-20 \mathrm{~Hz}$ signal. The lower panel shows the mean amplitude $( \pm 3 *$ SEM) of broadband for each Hilbert phase ( $\phi)$ of the $12-20 \mathrm{~Hz}$ signal. The dot-product of this mean with an exponential of the form $e^{i \phi}$ reveals the coupling phase and magnitude of the modulation $\left(Z_{\text {mod }}\right.$ - the portion of the $Z$-score of broadband power modulation with the phase of the $12-20 \mathrm{~Hz}$ rhythm). This calculation can be appreciated visually by taking the dot-product of mean broadband power with $\sin (\phi)$ and $\cos (\phi)$ independently, since $e^{i \phi}=\cos (\phi)+i \sin (\phi)$. (D) The "coupling vector" reveals the magnitude, $Z_{\text {mod' }}$ and phase, $\phi_{c^{\prime}}$ of the modulation of broadband activity by the $12-20 \mathrm{~Hz}$ rhythm in the complex plane. (E) The potential trace can be broken up into cue periods of visual search in different directions (colors) plus ISI periods (gray). (F) From each cue period, a single trial coupling vector can be obtained. (G) Plotting the distribution of coupling vectors across all cues reveals stronger coupling during ISI periods than during active visual search. $(\mathbf{H})$ For a given epoch type (in this case, ISI) a mean coupling vector (e.g., $\overline{Z_{\bmod }} * \mathrm{e}^{i \bar{\phi}}$ ) may be obtained. (I) The distribution of coupling values for each epoch can then be obtained by projecting their coupling vectors onto the epoch mean vector direction; for the $n$th trial, the magnitude of coupling contributing to the distribution of coupling values is $Z_{\text {mod }}(n)^{*} \cos (\varphi(n)-\bar{\varphi})$. Using the distribution of $Z_{\text {mod }}(n)$ alone biases the distribution to higher mean coupling values that would not be appropriate if there is no reproducible phase coupling. (J) Coupling strength for each cue type, projected onto its own mean coupling vector. The error bars indicate $3 *$ SEM. The error bars for visual search are typically larger than for ISI since there are four times more ISI cues than visual search cues in any particular direction. In this case, the coupling is strong and significant during ISI periods, and greatly reduced during all directions of visual search. and decrease of phase-amplitude modulation during visual search engagement suggests that rhythms can actively both suppress and facilitate sensory processing in the occipital cortex. Even though the spatial distribution of amplitude changes in rhythms can be widely distributed across the occipital lobe, the influence that they have on local cortical activity is gyrally specific.

\section{IMPLICATIONS OF RHYTHMIC MODULATION OF BROADBAND ACTIVITY}

From the perspective that broadband power reflects aggregate firing rate, the modulation of broad band activity by these lower frequency oscillations would indicate that these rhythms are influencing the instantaneous firing rate of many neurons simultaneously. This is consistent with coarse-graining of phenomena at the single-neuron scale, where the timing of individual action potentials is preferentially locked to a specific phase of ongoing local field potential oscillations ("spike-field coupling" - Buzsaki and Draguhn, 2004). Thus, ECoG rhythm phase to broadband amplitude findings, such as those reported in this study, may reveal a population-averaged reflection of this spike-field coupling. Because these experimental results imply that rhythms are modulating the activity of whole neuronal populations, a distinction must be drawn between fluctuations in rhythmic modulation and amplitude in a rhythm. For 

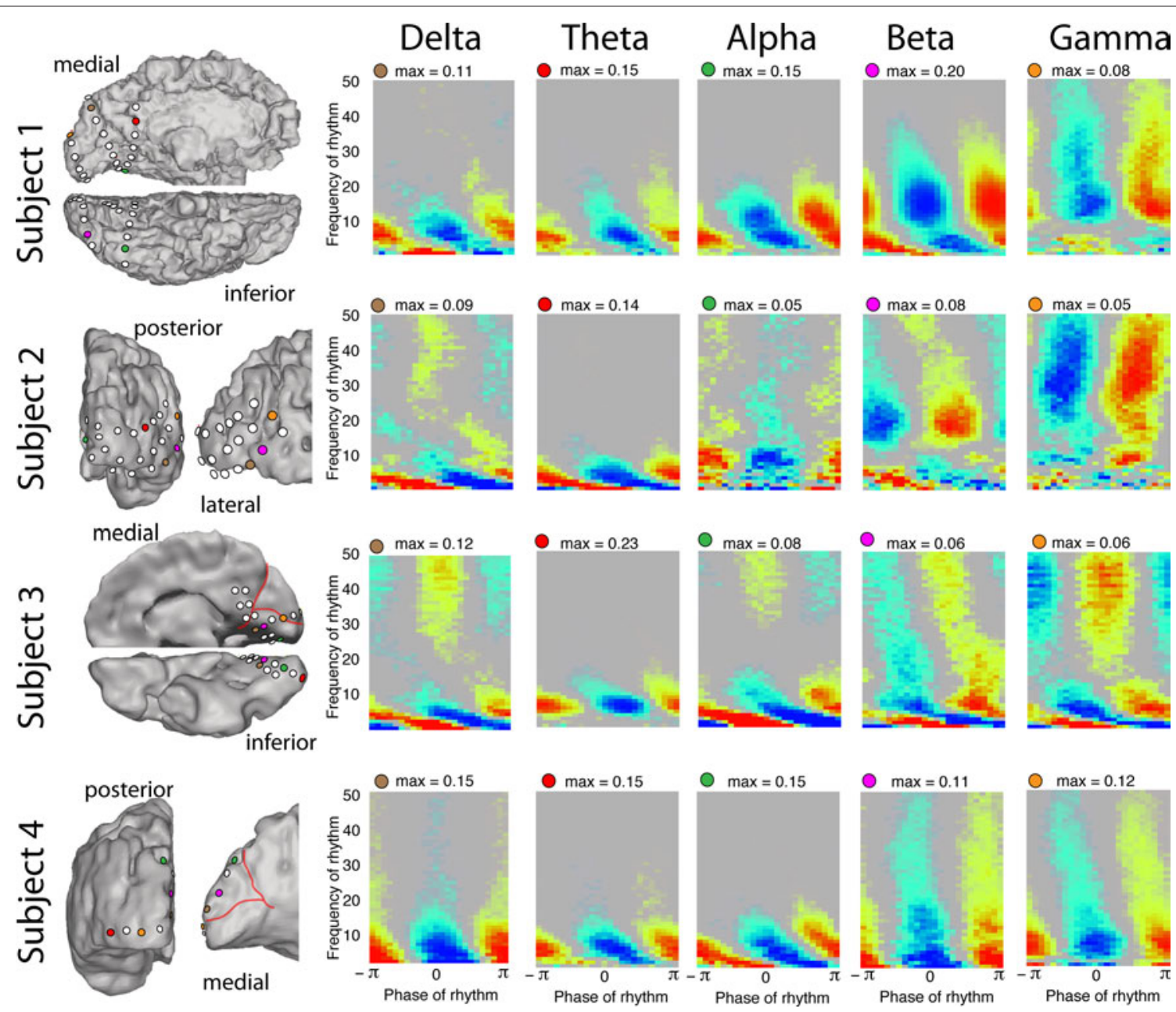

FIGURE 3 | Coupling to different rhythms in different subjects. Each row represents the palettes from five different electrode sites in subjects 1-4, respectively (site location color coded on the rendered brain surface). The palettes illustrate that local cortical activity (as revealed by broadband spectral change) is modulated by the $\delta / \theta / \alpha / \beta / \gamma$ rhythms in each case. Note that at many electrodes we observe coincident modulation of broadband power by multiple rhythms with distinct phases of preferred coupling. In subject 4, each of the palettes shown in the $\delta / \theta / \alpha$ coupling columns exhibit phase-amplitude coupling in all of those bands $(\delta / \theta / \alpha)$, and could each have been used to exemplify phase-amplitude coupling in any of the bands. All palettes for subject 5 are shown in Figure 4, where coupling to $\delta / \theta / \alpha / \beta / \gamma$ rhythms can also be observed. example, in Figure 4D there is a decrease in both the amplitude and the modulation associated with the 12-20 Hz rhythm, but in Figure 4G the decrease in $12-20 \mathrm{~Hz}$ rhythm is not accompanied by a corresponding decrease in modulation.

\section{TECHNICAL CONSIDERATIONS}

The techniques presented here for characterizing and statistically testing phase and amplitude interactions in the cortical surface potential are performed in stages, each of which is non-trivial. We independently isolate a broadband signal and simultaneous brain rhythms; subsequently, we examine correlations between these two phenomena. In order to test whether our assumptions about the underlying mechanisms could be valid, we constructed a simulated signal that had task-related broadband change, and was modulated by rhythmic influence in a task-dependent way (Figure 8). Our analytic algorithms were able to naively uncover the broadband change as well as the rhythmic modulation. These phenomena are not induced by event-related potentials; the ERP dies out after $400 \mathrm{~ms}$, and is thus too transient to account for the robust amplitude modulation we observe. Furthermore, the ISI periods had smaller or no ERPs, and exhibited dramatically larger modulation. Sharp discontinuities in the timeseries can give artificial phase modulation at higher frequencies, so artifactual epochs had to be excluded from analysis. When the method was applied to task-modulated brown noise (Figure 9), there was no coupling, suggesting that coupling is not a by-product of the method used for analysis.

\section{SUPPRESSION BY SYNCHRONIZATION}

Existing studies of rhythm-broadband modulations have mainly focused on a facilitatory role (Womelsdorf et al., 2005; Canolty et al., 2006; Jensen and Colgin, 2007; Siegel et al., 2008; He et al., 2010), suggesting that the presence of a rhythm enables an active component of local cortical processing. While this may be the case for 

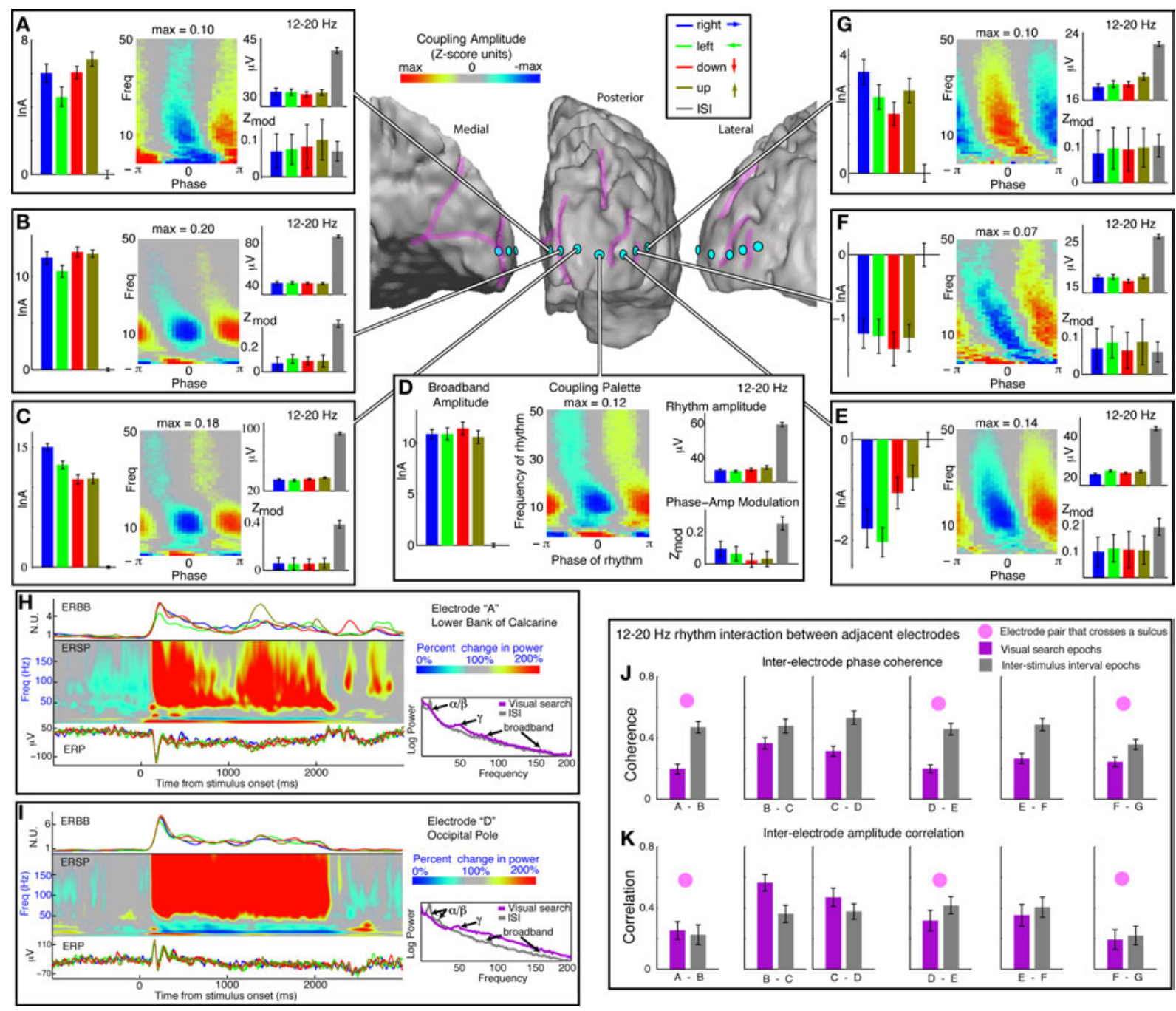

FIGURE 4 | Coupling across occipital cortex, in subject 5, with a focus on the $\boldsymbol{\beta}$-range (12-20 Hz). (A-G) Each of seven surface electrodes is shown on an electrode strip beginning in medial, peri-calcarine, occipital cortex, extending laterally around the occipital pole into the lateral occipital gyrus. The left-most series of bars in each panel is the log-broadband amplitude, in Z-score units, with the mean of ISI periods subtracted out. Errorbars represent $3 *$ SEM. The right-most error bars, flanking the horizontal origin, represent the variation during ISI periods. The medial 4 and the most lateral electrodes [e.g., (A-D), (G)] all show increased local cortical activity during visual search, while the 5th and 6th electrode sites show suppressed activity during visual search (these two share gyral location). The palettes show average coupling across all cues. All sites have strong and significant coupling to the $12-20 \mathrm{~Hz}(\beta)$ range. The upper-right axes within each panel show the amplitude of the $12-20 \mathrm{~Hz}$ rhythm during different cue types. There is a lower amplitude during visual search engagement in every case. The 12-20 Hz modulation of broadband amplitude, however, is selectively suppressed during task engagement in the (B)-(D) sites, which share common

$\theta$-range modulations, including those observed here, the $\beta$-range changes we observe are more suggestive of an inhibitory process in which the rhythm actively modulates local occipital cortical activity during periods of task disengagement (Handel et al.; Klimesch et al., 2007). In the setting of the $\gamma$-rhythm, it has been demonstrated that the influence of the rhythm facilitates cortical computation (Womelsdorf et al., 2007), particularly during visual processing.

gyral location. (H) Dynamics of visual response from the electrode highlighted in (A). On the Left:The upper trace is the average event-related broadband (ERBB) for each of the cue types. The middle plot is the average dynamic spectrum for all active sites combined called the "event-related spectral perturbation" (ERSP). The lower plot is the event-related potential (ERP) for each cue type. On the right, the changes in the mean spectrum during visual search (red) and ISI (blue) are shown. Note the so-called "event-related desynchronization" in the $\alpha / \beta$ rhythm range, and "event-related synchronization" in the $\gamma$-range (Pfurtscheller, 1999). Broadband change, though small, is present throughout - although it is covariant with the $\gamma$-rhythm change, it is a distinct entity. (I) Same analysis as in (H), for the electrode highlighted in (D). Broadband change is strong and robust, with $\alpha / \beta$-ERD and $\gamma$-ERS. In this case, the broadband change is large compared with the $\gamma$-rhythm ERS, although both can still be seen. The change at this site was representative of all three occipital pole electrodes - sites (B)-(D). (J) Inter-electrode phase coherence in the $12-20 \mathrm{~Hz}$ range. (K) Inter-electrode amplitude correlation in the $12-20 \mathrm{~Hz}$ range.

These observations have been linked to the hypothesis that rhythms enhance spike transmission between synchronized areas - "communication through coherence" (Singer, 1993; Fries, 2005). This is consistent with the increase in power that we observe in $\gamma$-rhythms (Figures $4 \mathrm{H}, \mathbf{I}$ ). We propose that rhythms may also play a role in suppressing local cortical computation, with the cortically suppressed (disengaged) state one in which widespread populations 


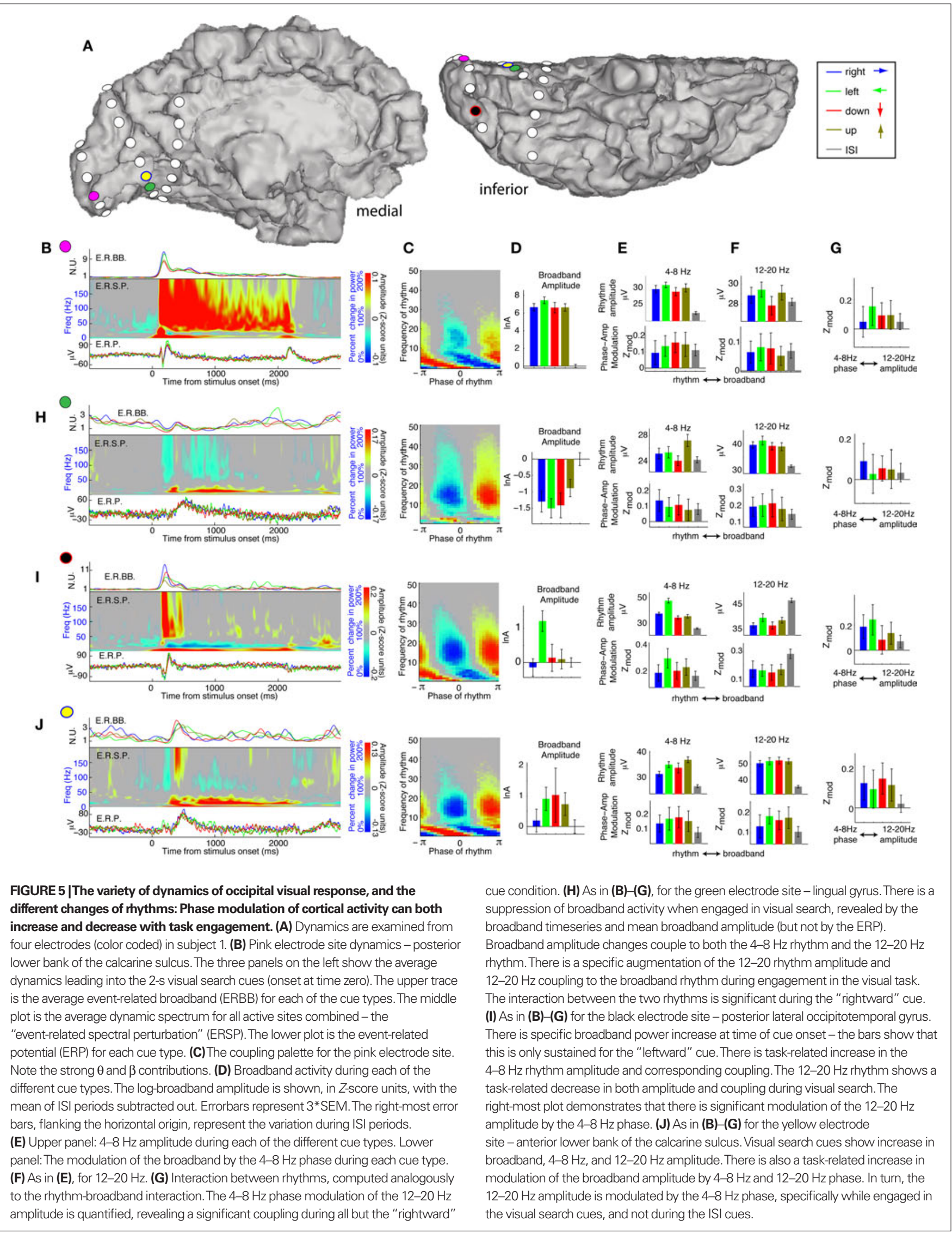




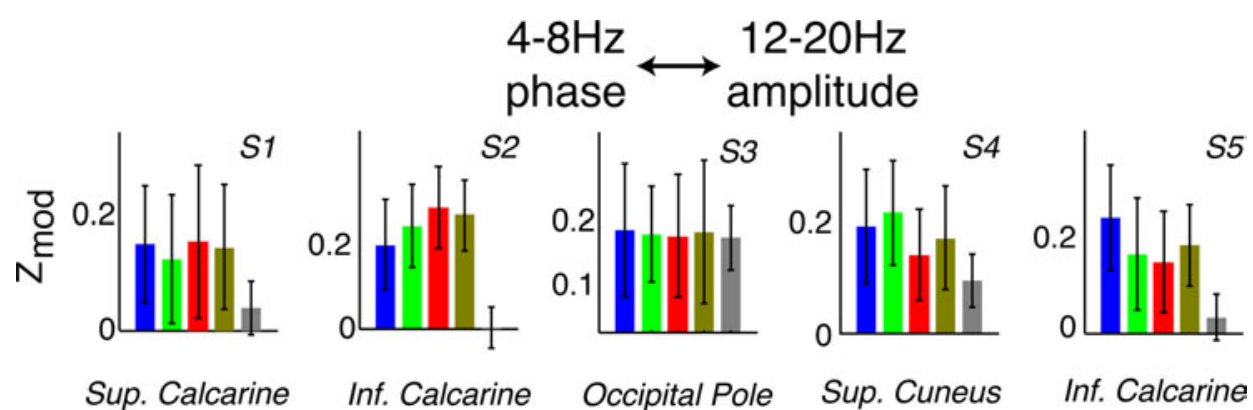

FIGURE 6 |The rhythms interact with one another. Examples of 4-8 Hz phase modulation of 12-20 Hz amplitude in all five subjects (denoted S\#). Note that there are examples of this kind of coupling in each subject, but most electrodes did not show significant phase-amplitude relationships between rhythms.

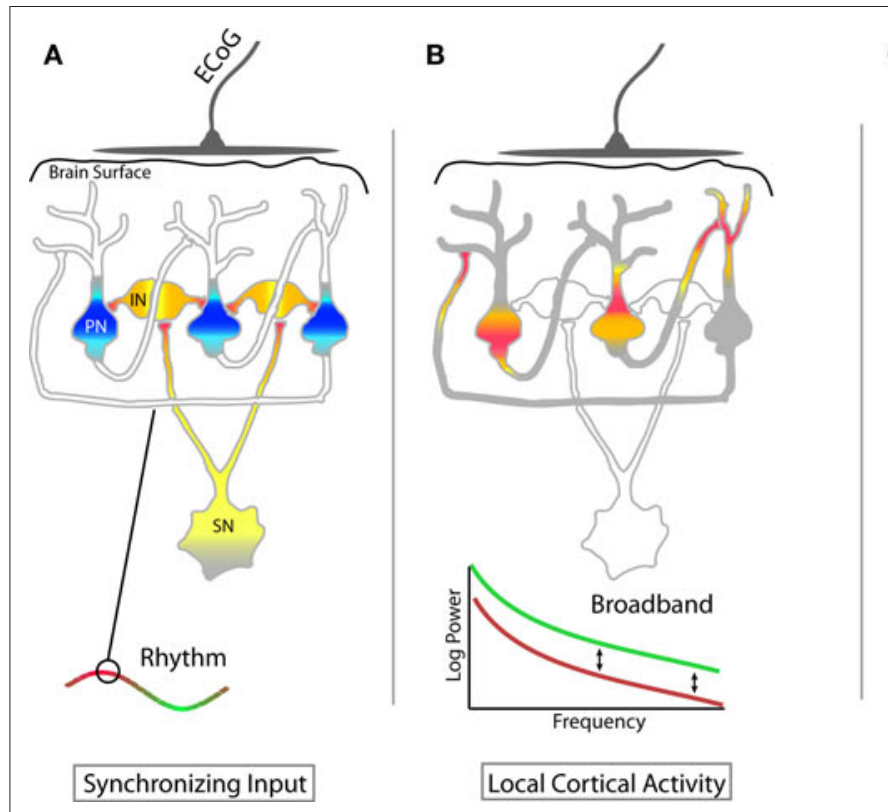

FIGURE 7 | Heuristic for inhibition through synchronization. While there are many potential roles subserved by different rhythms, one potential role that we highlight is inhibition of population-scale processing by synchronization. This inhibitory function for rhythms is supported in cases where there is a clear, robust decrease in phase modulation of broadband by the rhythms' phase during visual task engagement (e.g., Figures 4B-E and 5H). (A) Input from a "synchronizing neuron" (SN) projects diffusely to an inhibitory population of layer $4 / 5$ neurons (IN) which in concert inhibit pyramidal neurons (PN) at their basal dendrites and soma. When averaged across the cortex immediately beneath an ECoG electrode, this is revealed by the presence of a rhythm. (yellow/orange/red depolarization; light/dark blue - hyperpolarization). When present, the rhythm

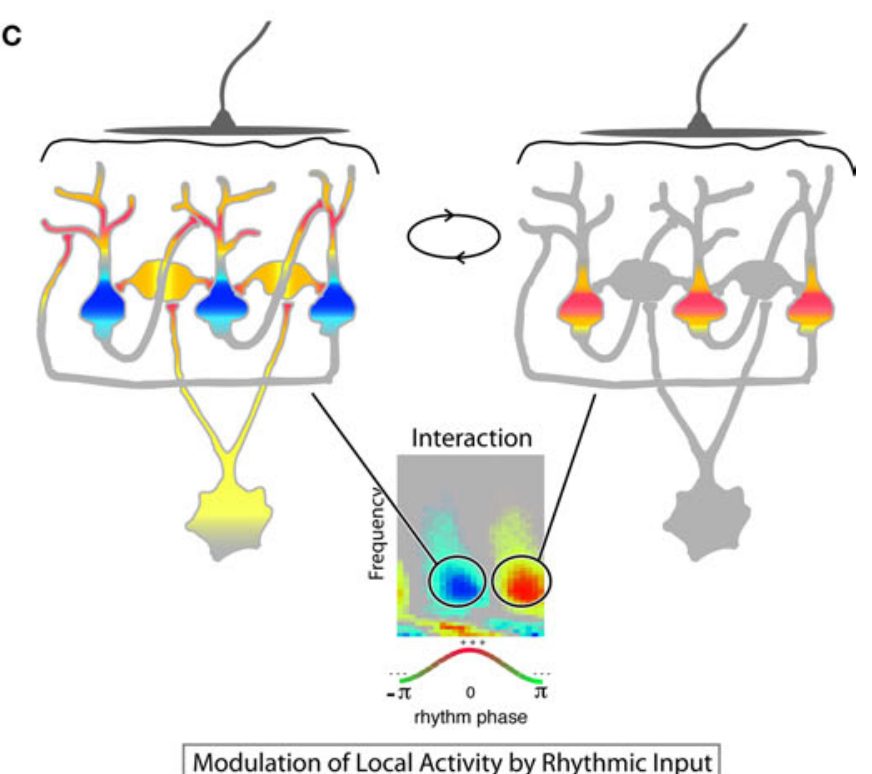

keeps the cortex in an idling, dynamically "ready," state - shut down, but rapidly able to shift to active computation. Blanket inhibition, could also prevent computation, but would not allow for easy transition to diverse and complex patterns of activity. (B) During active computation, the population of pyramidal neurons engages in a complex pattern of mutual excitation. Averaged across the population, these appear Poisson-distributed, and no special timescale emerges in the ECoG signal produced by this local, cortical-cortical interaction. (C) The synchronized influence of the rhythm forces cortical-cortical inputs to synchronize, effectively reducing their ability to perform computation. This modulation of local activity by rhythms is revealed at our ECOG scale by significant broadband modulation by low-frequency phase. of cortical neurons are phase-coupled to the rhythm (Figure 7). In this "suppression-by-synchronization" model, neurons from a distant "pacemaker" circuit project diffusely to populations of cortical pyramidal neurons, targeting their basal dendrites and somas with synchronized input. Whether the synchronized inputs are excitatory or inhibitory, the cortico-cortical inputs between pyramidal neurons will need to be stochastically resonant with the synchronized input to induce a downstream action potential (anti-aligned if the synchronizing input is inhibitory, aligned if it is excitatory). In this way, weak but synchronous input keeps the population in a "dynamically suppressed" state, where it can quickly transition into an engaged "processing state." An alternative model - suppression by blanket inhibition, would be more metabolically expensive and would also not allow for easy transition from the suppressed state to a computing state because the targeted pyramidal population would have to overcome an effective hyperpolarization barrier. In the suppression-by-synchronization regime, one need only remove time-locking influence and the neuronal population can switch to an engaged and actively computing state. At this stage, identifying the anatomic locus of the synchronizing source will require further 


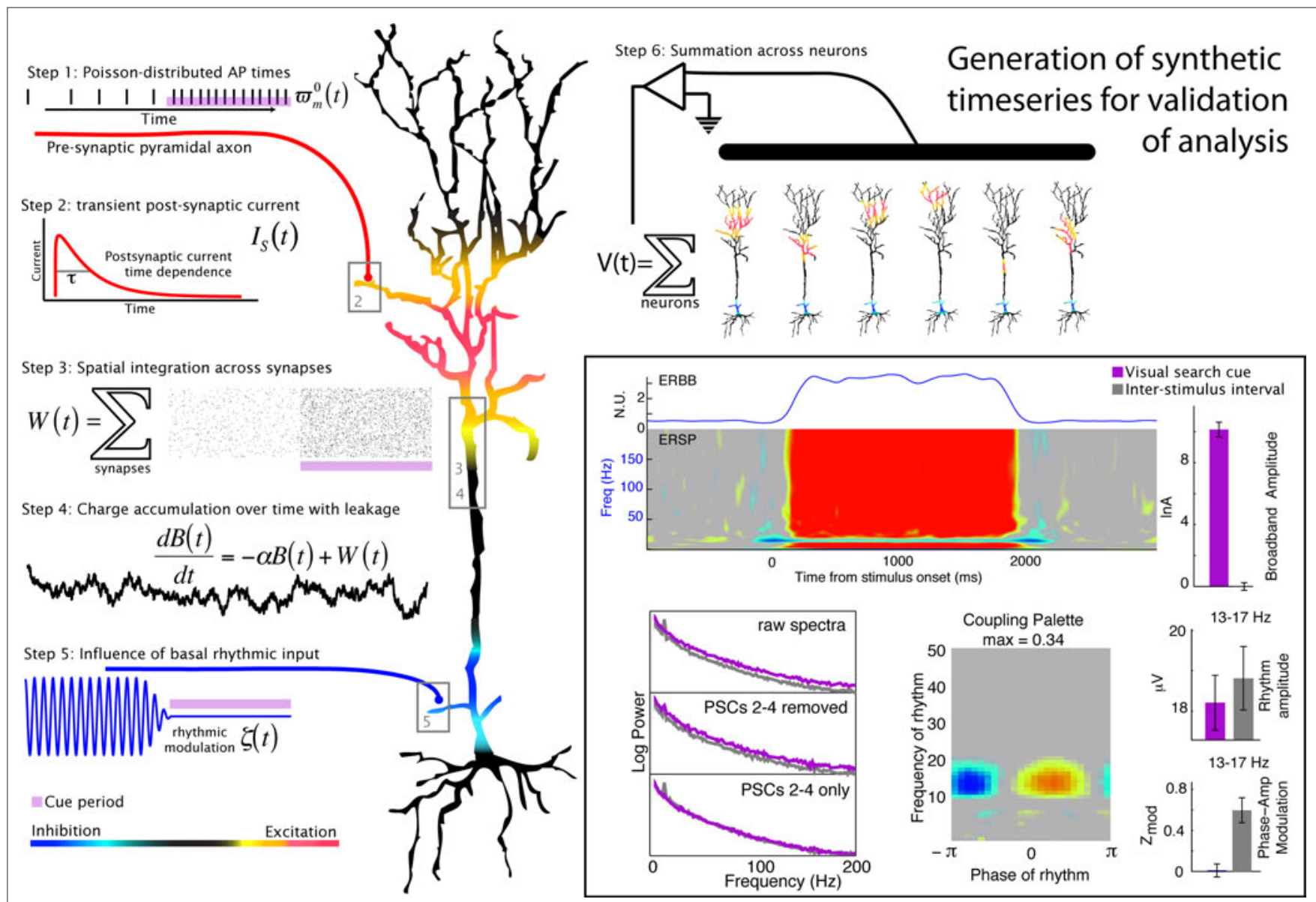

FIGURE 8 | Generation of a simulated timeseries and validation of signal processing technique. The illustrated heuristic was used to generate a $1 / f$ base, broadband, synthetic timeseries (e.g., Miller et al., 2009b) that is modulated by a $15 \mathrm{~Hz}$ rhythm (described in detail in the Appendix). In the boxed region on the bottom right, the synthetic timeseries is analyzed in the same way as the physiological data. It demonstrates that the decoupling and phase-modulation estimation signal processing techniques described in this manuscript work for simulated data that were based on the mechanisms hypothesized for our physiological data (e.g., Figure 7).
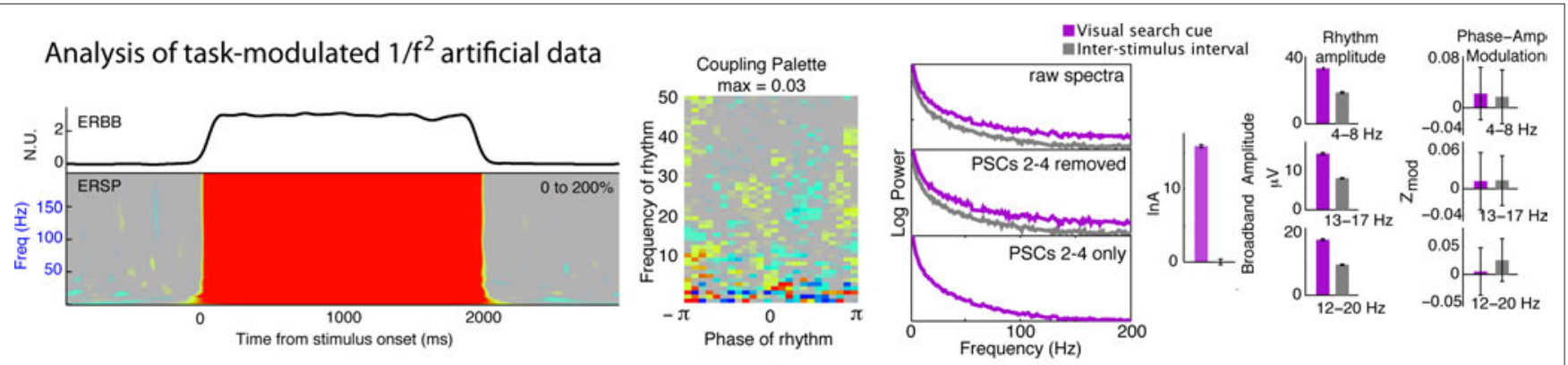

FIGURE 9 |Application of signal processing technique to colored noise. When the signal processing methods were applied to task-modulated $P \sim f^{2}$ colored noise as a negative control, there is no significant coupling.

research. The thalamus, specifically the lateral pulvinar and lateral geniculate nucleus, is a compelling candidate because of its diffuse projection and known role in visual processing (Zhang et al., 2010). Additionally, it has been demonstrated that weak but synchronous thalamic input can drive cortical processing dramatically in the active feedforward state (Bruno and Sakmann, 2006). Whatever the case, this rhythmic modulatory influence acts over large cortical regions, and coherence is significantly decreased during visual engagement (Figure 4J). Perhaps the same is true for selective suppression of other cortical regions. During behavior, some rhythms 
might serve as "spotlights of utility" that, at baseline, actively suppress non-relevant cortical areas by synchronization. When the cortical area becomes functionally relevant the rhythm is withdrawn.

\section{UNIVERSAL RHYTHMIC MOTIFS?}

Although it is frequently treated as if a rhythm at a particular frequency has a universal functional property, a rhythm with a particular center frequency need not serve a single neural function in every state and location in which it is observed. The fact that we observe different preferred phases, and also conjugate task-related changes in the same frequency ranges, in different cortical areas, suggests that there is unlikely to be a simple, universal, role for a particular band-limited rhythm. What we call " $\beta$ " in the lateral occipital gyrus and what we call " $\beta$ " at the occipital pole may in fact represent different phenomena, with different physiological origins, but with a common timescale and corresponding frequency range (Figure 4). That said, the observation of significant changes in inter-electrode coherence between adjacent electrodes suggests a common etiology that selectively releases some cortical areas (e.g., sites Figures $\mathbf{4 A - D , G}$ ) but not others (sites Figures $4 \mathbf{E}, \mathbf{F}$ ), in a gyrally-conserved way, during task engagement.

The fact that the phase-amplitude coupling motifs sometimes shift substantially in phase across subjects or electrodes might point to different types of physiologic phenomena which are revealed with different types of rhythmic coupling motifs. For example, a rhythm with one preferred phase might exert an excitatory influence via one class of channels, and a different rhythm might exert an inhibitory influence via a separate class of channels. The different timescales implied by the frequencies different rhythmic phenomena might reflect feedback loops involving different numbers of neurons, or slow versus fast types of membrane channels. Some rhythms might reflect emergent properties of highly interconnected networks, while other rhythms of the same frequency range but different cortical location, might reflect cortical-subcortical feedback loops.

\section{REFERENCES}

Beck, A. (1891). Mapping of localization in brain and cortex by means of electric phenomena. Polska Akademica Umiejetnosci 2, 186-232.

Bedard, C., Kroeger, H., and Destexhe, A. (2006). Does the $1 / \mathrm{f}$ frequency scaling of brain signals reflect self-organized critical states? Phys. Rev. Lett. 97, 118102.

Brindley, G. S., and Craggs, M. D. (1972). The electrical activity in the motor cortex that accompanies voluntary movement. J. Physiol. (Lond.) 223, 28P.

Bruno, R. M., and Sakmann, B. (2006). Cortex is driven by weak but synchronously active thalamocortical synapses. Science 312, 1622.

Buzsaki, G., and Draguhn, A. (2004). Neuronal oscillations in cortical networks. Science 304, 1926.

Canolty, R. T., Edwards, E., Dalal, S. S., Soltani, M., Nagarajan, S. S., Kirsch, H. E., Berger, M. S., Barbaro, N. M., and Knight, R. T. (2006). High gamma

The observed nesting of $12-20 \mathrm{~Hz}$ amplitude in the $4-8 \mathrm{~Hz}$ phase demonstrates that, in some cases, the physiologic processes underlying these rhythms can interact with one another, and in a task-dependent manner (see, for example Lakatos et al., 2005; Tort et al., 2009).

\section{EMERGING QUESTIONS}

This study is part of a continuing project to investigate the many phase-phase, phase-amplitude and amplitude-amplitude couplings both within and between electrodes on the cortical surface. Are there functionally relevant shifts in coupling phase across distinct behavioral states? Are these interactions present between cortical regions communicating over many centimeters? What rhythms play a role in different cognitive and sensorimotor processes? Can we measure a distant source of the rhythm at the same time as the nested oscillation, and is there in fact such a source? Is there a direct relationship between the phase of the coupling we observe and the spike-field coupling measured at the single-neuron scale? Answers to these questions will likely pave the way for a new understanding of large-scale information processing mechanisms in the human brain.

\section{ACKNOWLEDGMENTS}

We appreciate the time and dedication of the patients and staff at Harborview Hospital in Seattle, WA. We thank Jaan Aru and Lise Johnson for helpful discussion. This research was supported by generous the contribution of National Aeronautics and Space Administration Graduate Student Research Program (KJM); National Institute of General Medical Sciences Medical Scientist Training Program (KJM); National Science Foundation 0622252 and 0642848 (KJM, RPNR, JGO); National Institute of Health R21-DA024423 (CJH), RO1-NS12542 (EEF), R01NS065186 (KJM, JGO); James S. McDonnell fund - 223921 26239B (ECL).

Fries, P. (2005).A mechanism for cognitive dynamics: neuronal communication through neuronal coherence. Trends Cogn. Sci. (Regul. Ed.) 9, 474-480.

Fries, P., Reynolds, J. H., Rorie, A. E., and Desimone, R. (2001). Modulation of oscillatory neuronal synchronization by selective visual attention. Science 291, 1560.

Goupillaud, P., Grossmann, A., and Morlet, J. (1984). Cycle-octave and related transforms in seismic signal analysis. Geoexploration 23, 85-102.

Handel, B. F., Haarmeier, T., Jensen, O. Alpha oscillations correlate with the successful inhibition of unattended stimuli.J. Cogn. Neurosci. [Epub ahead of print].

He, B. J., Zempel, J. M., Snyder, A. Z., and Raichle, M. E. (2010). The temporal structures and functional significance of scale-free brain activity. Neuron 66, 353-369.

Hermes, D., Miller, K. J., Noordmans, H. J., Vansteensel, M. J., and Ramsey, N. F. (2010). Automated electrocor- ticographic electrode localization on individually rendered brain surfaces. $J$. Neurosci. Methods 185, 293-298.

Jacobs, J., and Kahana, M. J. (2010). Direct brain recordings fuel advances in cognitive electrophysiology. Trends Cogn. Sci. (Regul. Ed.) 14, 162-171.

Jensen, O., and Colgin, L. L. (2007). Crossfrequency coupling between neuronal oscillations. Trends Cogn. Sci. (Regul. Ed.) 11, 267-269.

Jolliffe, I. T. (2002). Principal Component Analysis. Heidelberg: Springer Verlag.

Klimesch, W., Sauseng, P., and Hanslmayr, S. (2007). EEG alpha oscillations: the inhibition-timing hypothesis. Brain Res. Rev. 53, 63-88.

Lakatos, P., Shah, A. S., Knuth, K. H., Ulbert, I., Karmos, G., and Schroeder, C. E. (2005). An oscillatory hierarchy controlling neuronal excitability and stimulus processing in the auditory cortex. J. Neurophysiol. 94, 1904.

Lindén, H., Pettersen, K. H., and Einevoll, G.T.(2010).Intrinsic dendritic filtering gives low-pass power spectra of local 
field potentials. J. Comput. Neurosci. 1-22. [Epub ahead of print].

Makeig, S., Westerfield, M., Jung, T. P., Enghoff, S., Townsend, J., Courchesne, E., and Sejnowski, T. J. (2002). Dynamic brain sources of visual evoked responses. Science 295, 690.

Manning, J. R., Jacobs, J., Fried, I., and Kahana, M.J. (2009). Broadband shifts in local field potential power spectra are correlated with single-neuron spiking in humans. J. Neurosci. 29, 13613.

Miller, K. J. (2010). Broadband spectral change: evidence for a macroscale correlate of population firing rate? J. Neurosci. 30, 6477.

Miller, K. J., Weaver, K. E., and Ojemann, J. G. (2009a). Direct electrophysiological measurement of human default network areas. Proc. Natl. Acad. Sci. U.S.A. 106, 12174.

Miller, K. J., Sorensen, L. B., Ojemann, J. G., and den Nijs, M. (2009b). Powerlaw scaling in the brain surface electric potential. PLoS Comput. Biol. 5, e1000609. doi: 10.1371/journal. pcbi.1000609.

Miller, K. J., Zanos, S., Fetz, E. E., den Nijs, M., and Ojemann, J. G. (2009c). Decoupling the cortical power spectrum reveals real-time representation of individual finger movements in humans. J. Neurosci. 29, 3132.

Miller, K. J., Schalk, G., Fetz, E. E., Den Nijs, M., Ojemann, J. G., and Rao, R. P. N. (2010a). Cortical activity during motor execution, motor imagery, and imagery-based online feedback.
Proc. Natl. Acad. Sci. U.S.A. 108, 4430-4435.

Miller, K. J., Hebb, A. O., Hermes, D., den Nijs, M., Ojemann, J. G., and Rao, R. P. N. (2010b). Brain surface electrode co-registration using MRI and X-ray. IEEE Eng. Med. Biol. Soc. (in print).

Mitchell, J. F., Sundberg, K. A., and Reynolds, J. H. (2009). Spatial attention decorrelates intrinsic activity fluctuations in macaque area V4. Neuron 63, 879-888.

Mormann, F., Fell, J., Axmacher, N., Weber, B., Lehnertz, K., Elger, C. E., and Fernández, G. (2005). Phase/ amplitude reset and theta-gamma interaction in the human medial temporal lobe during a continuous word recognition memory task. Hippocampus 15, 890-900.

Okun, M., Naim, A., and Lampl, I. (2010). The subthreshold relation between cortical local field potential and neuronal firing unveiled by intracellular recordings in awake rats. J. Neurosci. 30, 4440 .

Osipova, D., Hermes, D., and Jensen, O. (2008). Gamma power is phaselocked to posterior alpha activity. PLoS ONE 3, e3990. doi: 10.1371/journal. pone. 0003990 .

Penny, W. D., Duzel, E., Miller, K. J., and Ojemann, J. G. (2008). Testing for nested oscillation.J. Neurosci. Methods 174, 50-61.

Pfurtscheller, G. (1999). Event-Related Desynchronization (ERD) and Event
Related Synchronization (ERS). Baltimore: Williams and Wilkins.

Porat, B. (1997). A Course in Digital Signal Processing. New York: Wiley.

Sabatini, B. L., and Regehr, W. G. (1996). Timing of neurotransmission at fast synapses in the mammalian brain. Nature 384, 170-172.

Siegel, M., Donner, T. H., Oostenveld, R., Fries, P., and Engel, A. K. (2008). Neuronal synchronization along the dorsal visual pathway reflects the focus of spatial attention. Neuron 60 , 709-719.

Singer, W. (1993). Synchronization of cortical activity and its putative role in information processing and learning. Annu. Rev. Physiol. 55, 349-374.

Tort, A. B. L., Komorowski, R. W., Manns, J. R., Kopell, N. J., and Eichenbaum, H. (2009). Theta-gamma coupling increases during the learning of itemcontext associations. Proc. Natl. Acad. Sci. U.S.A. 106, 20942.

Whittingstall, K., and Logothetis, N. K. (2009). Frequency-band coupling in surface EEG reflects spiking activity in monkey visual cortex. Neuron 64, 281-289.

Womelsdorf, T., Fries, P., Mitra, P. P., and Desimone, R. (2005). Gamma-band synchronization in visual cortex predicts speed of change detection. Nature 439, 733-736.

Womelsdorf, T., Schoffelen, J. M., Oostenveld, R., Singer, W., Desimone, R., Engel, A. K., and Fries, P. (2007). Modulation of neuronal interactions through neuronal synchronization. Science 316, 1609.

Zhang, D., Snyder, A. Z., Shimony, J. S., Fox, M. D., and Raichle, M. E. (2010). Noninvasive functional and structural connectivity mapping of the human thalamocortical system. Cereb. Cortex 20, 1187-1194.

Conflict of Interest Statement: The authors declare that the research was conducted in the absence of any commercial or financial relationships that could be construed as a potential conflict of interest.

Received: 26 July 2010; accepted: 29 September 2010; published online: 29 October 2010.

Citation: Miller KJ, Hermes D, Honey CJ, Sharma M, Rao RPN, den Nijs M, Fetz EE, Sejnowski TJ, Hebb AO, Ojemann JG, Makeig S and Leuthardt EC (2010) Dynamic modulation of local population activity by rhythm phase in human occipital cortex during a visual search task. Front. Hum. Neurosci. 4:197. doi: 10.3389/ fnhum.2010.00197

Copyright ( 2010 Miller, Hermes, Honey, Sharma, Rao, den Nijs, Fetz, Sejnowski, Hebb, Ojemann, Makeig and Leuthardt. This is an open-access article subject to an exclusive license agreement between the authors and the Frontiers Research Foundation, which permits unrestricted use, distribution, and reproduction in any medium, provided the original authors and source are credited. 


\section{METHODOLOGICAL APPENDIX SIGNAL PRE-PROCESSING}

To reduce common artifact, the potential, $V_{n}^{0}(t)$, measured at each electrode $n$ was re-referenced with respect to the common average of all electrodes, $V_{n}(t)=V_{n}^{0}(t)-1 / N \sum_{i=1}^{N} V_{i}^{0}(t)$, (channel label, $n$, henceforth dropped). Electrodes with significant artifact or epileptiform activity were rejected prior to common averaging. Epochs that appeared artifactual were rejected. Following rereferencing, only occipital electrodes were further examined; the remainder was discarded. Ambient line noise was rejected by notch filtering between 58 to $62 \mathrm{~Hz}$ using a 3rd-order Butterworth filter (Porat, 1997).

\section{POWER SPECTRAL DENSITIES}

\section{Power spectral snapshots}

A set of epochs surrounding onset and middle of each visual search and ISI cue, $\tau$, were extracted from $V(t)$; each epoch was of duration $T=1 \mathrm{~s},\left(\tau_{q}-(1 / 2) T\right)<t<\left(\tau_{q}+(1 / 2) T\right)$. The epochs were sorted according to cue type $q$, and labeled by their event markers $\tau_{q}$ [note times of onset, middle of both cues and ISI]. The power spectral density (PSD) of the epoch flanking time $\tau_{q}$ was calculated as:

$P(f, q)=\left|\frac{1}{\sqrt{T}} \sum_{t=-T / 2}^{+T / 2} V\left(\tau_{q}+t\right) H(t) e^{i 2 \pi f t}\right|^{2}$

with Hann window (Porat, 1997)

$H(t)=\frac{1}{2}\left(1-\cos \left(\frac{2 \pi t}{T}\right)\right)$.

\section{DYNAMIC POWER SPECTRAL MEASURES}

Time-frequency approximations (dynamic spectra) were made using both a wavelet and a Hilbert transform approach.

\section{Wavelet approach}

A Morlet wavelet (Goupillaud et al., 1984) of the form: $\psi(\tau, t)=\exp (i 2 \pi t / \tau) \exp \left(-t^{2} / 2 \tau^{2}\right)$ was convolved with the timeseries to get a time-frequency estimate for every $f=1 / \tau$ :

$\tilde{V}(1 / \tau, t)=\sum_{t^{\prime}=-5 \tau / 2}^{5 \tau / 2} V\left(t+t^{\prime}\right) \psi\left(t^{\prime}, \tau\right)$

A total of 5 cycles $(5 \tau)$ was used to estimate the amplitude and phase of the signal at each frequency for every point in time. In this way a time-varying Fourier component $\tilde{V}(f, t)=r(f, t) \mathrm{e}^{i \varphi(f, t)}$, with fixed uncertainty between the confidence in the estimate of the instantaneous amplitude and phase versus the confidence in temporal resolution is obtained at each $\mathrm{Hz}$.

\section{Hilbert transform approach}

A complex signal to reflect the timecourse of a functionally relevant frequency-range band was constructed as follows: The signal $V(t)$ was band-passed using a 3rd-order Butterworth filter for a specific range, to obtain the "band-limited" potential, $V(F, t)$, where $F$ denotes the frequency range (e.g., for "alpha," $F \rightarrow[8-12 \mathrm{~Hz}]$, etc.). A complex analytic signal, $\tilde{V}(F, t)=V(F, t)+i V^{I M}(F, t)$ was constructed using the Hilbert transform (e.g., such that the new signal satisfies the Cauchy-Riemann conditions for analyticity at all times).

\section{Discrete-time signal processing}

This signal may also be expressed in polar notation: $\tilde{V}(F, t)=$ $r(F, t) \mathrm{e}^{i \phi(F, t)}$. The "analytic amplitude" of the range $F$ at time $t$ is $r(F$, $t$ ) and the "phase" is $\phi(F, t)$. The interpretation of $\phi$ is intuitively difficult, but the most concrete understanding is that the rhythm captured by range $F$ is most surface-positive at $\phi=0$, and most surface-negative at $\phi=\pi$ or $-\pi$. Note that $\phi$ becomes poorly defined as $r \rightarrow 0$. For discussion of different approaches and illustration of nested ECoG measurements, see, for example, Penny et al. (2008).

\section{AMPLITUDE CORRELATION AND PHASE COHERENCE OF RHYTHMS}

The relationships between complex rhythms $\tilde{V}_{n}(F, t)=r_{n}(F, t) \mathrm{e}^{i \phi_{n}(F, t)}$ for trial $k$ (electrode $n$ ) were determined as follows. The inter-electrode amplitude correlation $R_{a b}(k)$ for the rhythm defined by range of frequencies $F$ between electrodes $a$ and $b$, during trial $k$ is:

$R_{a b}(k)=\frac{1}{T_{k}} \sum_{t_{k} \in k} r_{a}^{z}\left(F, t_{k}\right) r_{b}^{z}\left(F, t_{k}\right)$

Where $t_{k}$ are the times of epoch $k$ ( $t_{k} \in k$, total $T_{k}$ timepoints) and the $z$-score amplitude for the epoch is:

$$
\begin{aligned}
& r_{n}^{z}\left(F, t_{k}\right)=\frac{r_{n}\left(F, t_{k}\right)-\left\langle r_{n}\right\rangle_{t_{k} \in k}}{\left.\sigma_{r_{n}}\right|_{t_{k} \in k}}, \text { where } \\
& \left\langle r_{n}\right\rangle_{t_{k} \in k}=\frac{1}{T_{k}} \sum_{t_{k} \in k} r_{n}\left(F, t_{k}\right), \\
& \left.\sigma_{r_{n}}\right|_{t_{k} \in k}=\left(\frac{1}{T_{k}} \sum_{t_{k} \in k}\left(r_{n}\left(F, t_{k}\right)-\left\langle r_{n}\right\rangle_{t_{k} \in k}\right)^{2}\right)^{1 / 2}
\end{aligned}
$$

The single trial inter-electrode phase coherence, $Q_{a b}(k)$, is:

$Q_{a b}(k)=\left|\frac{1}{T_{k}} \sum_{t_{k} \in k} \mathrm{e}^{i\left(\phi_{a}(F, t)-\phi_{b}(F, t)\right)}\right|$

\section{STIMULUS-TRIGGERED AVERAGE OF TIME-FREQUENCY POWER ESTIMATE}

This time-frequency approximation can be used to calculate mean power in relation to the onset of visual stimuli:

$$
\bar{P}^{v}\left(f, t_{w}\right)=\frac{1}{N_{v}} \sum_{\tau_{v}}\left(\frac{\left|\tilde{V}\left(f, t_{w}+\tau_{v}\right)\right|^{2}}{\frac{1}{N_{t^{\prime}}} \sum_{t^{\prime}}\left|\tilde{V}\left(f, t^{\prime}\right)\right|^{2}}\right)
$$

Where $\tau_{v}$ denote onset times of visual search cues (total $N_{v}$ ), and $t^{\prime}$ denote inter-stimulus times (total $N_{t^{\prime}}$ ). The peristimulus time window of interest is denoted $t_{w}$; in our case, $-1 s<t_{w} \leq 3 s$. These normalized maps of power as a function of time and frequency provide important information about characteristic spectral changes with local cortical function (also called "event-related spectral perturbations" - ERSPs; Makeig et al., 2002). While ERSPs could be 
calculated independently for each condition (arrow direction) of the visual search task, for simplicity we calculate an ERSP collapsed across conditions.

\section{DECOUPLING THE CORTICAL SPECTRUM TO SEPARATE RHYTHMIC ACTIVITY AWAY FROM BROADBAND CHANGE}

The decoupling process is described and illustrated in detail in the main text and supplement to Miller et al. (2009c). It was applied here as follows:

\section{Principal component decomposition of spectral change}

The samples of the PSD, $P(f, q)$, (total $\left.N_{q}\right)$, were normalized prior to decomposition.

$\widehat{P}(f, q)=\ln (P(f, q))-\ln \left(\frac{1}{N_{q}} \sum_{q} P(f, q)\right)$

The PCA method (Jolliffe, 2002) determines the eigenvalues $\lambda_{k}$ and eigenvectors $\overrightarrow{\mathrm{e}}_{k}$ of the correlation matrix:

$C\left(f, f^{\prime}\right)=\sum_{q} \hat{P}(f, q) \hat{P}\left(f^{\prime}, q\right)$

These eigenvectors, $\hat{C} \overrightarrow{\mathrm{e}}_{k}=\lambda_{k} \overrightarrow{\mathrm{e}}_{k}$, the "Principal Spectral Components" (PSCs), reveal which frequencies vary together, and are ordered by magnitude of corresponding eigenvalue: $\lambda_{1}>\lambda_{2}>\cdots>\lambda_{N_{f}}$ ( $N_{f} \equiv$ number of frequencies). If we define the rotation matrix $A(f, k)=\left(\overrightarrow{\mathrm{e}}_{1}, \overrightarrow{\mathrm{e}}_{2}, \cdots, \overrightarrow{\mathrm{e}}_{N_{f}}\right)$, then the projection, $W(k, q)$, of each individual original spectrum in the ensemble onto the new basis vector $k$ is

$W(k, q)=\sum_{f} A(k, f) \hat{P}(f, q)$.

The inverse rotation matrix $\hat{A}^{-1}, \hat{A}^{-1} \hat{A}=\hat{I}$, allows us to compare and visualize specific subsets of PSC components with the original full spectrum in frequency space. The 2nd to 4th PSCs typically capture rhythmic power spectral phenomena, and power spectra can be reconstructed with and without this rhythmic influence:

$\widehat{P}_{\Xi}(f, q)=\sum_{k \in \Xi} A^{-1}(f, k) W(k, q)$.

If the 2 nd to 4 th PSCs are omitted, $\Xi \rightarrow\left\{1,5 \ldots N_{f}\right\}$, then PSDs can be reconstructed where changes in rhythmic spectral phenomena are mostly removed (although there may be residual variance in the decomposition, or some rhythmic influence in all cases). If $\Xi \rightarrow\{2-4\}$, then PSDs can be reconstructed where changes in rhythmic spectral phenomena are mostly isolated (Figures 1C and 7).

\section{The timecourse of broadband spectral change}

The time-dependent, normalized, dynamic spectrum, $\widehat{P}(f, q)$, can be obtained in parallel fashion to the spectral snapshots.

$$
\begin{aligned}
& P(f, t)=\frac{|\tilde{V}(f, t)|^{2}}{\frac{1}{N_{t}} \sum_{t}|\tilde{V}(f, t)|^{2}}, \\
& \hat{P}(f, t)=\ln (P(f, t))-\ln \left(\frac{1}{N_{t}} \sum_{t} P(f, t)\right) .
\end{aligned}
$$

The reflection of the 1st PSC $\left(\overrightarrow{\mathrm{e}}_{1}\right)$ in the dynamic spectrum can be estimated by projecting the dynamic spectrum onto it.

$$
\ln A(t)=\sum_{f} \mathrm{e}_{1}(f) \hat{P}(f, t)
$$

We call it $\ln A(t)$ here, because it approximates the logarithm of the timecourse of the coefficient of a power-law in the cortical spectrum of the form $P(f, t)=A(t) f^{-x}$ (Miller et al., 2009b); it is smoothed with a Gaussian window of $50 \mathrm{~ms}$ standard deviation, $z$-scored, and exponentiated to obtain the "broadband" traces of Figures 1D, 4H,I and 5B,H-J (e.g., time-varying estimates of the coefficient of the power-law spectrum). The broadband power timecourses are robust estimates of behaviorally relevant local cortical activity (Miller et al., 2009c). Because the quantity $\ln A(t)$ is approximately log-normal distributed, we express it in $z$-score units, and, for notational brevity, denote it $\chi(t)$ in connection to the broadband power-law it reflects.

\section{SYNTHETIC DATA (FIGURE 8)}

The heuristic method illustrated in Figures 7A-C was used to create simulated data by combining input signals whose properties are known. The synthetic data could then be analyzed to validate the methods developed for the cortical data. The process is an extension of the one used to illustrate broadband, power-law, spectral change in previous manuscripts (Bedard et al., 2006; Miller et al., 2009b; Miller, 2010). Recent in vivo simultaneous recordings have demonstrated a strong correlation between trans-membrane and local field potentials (Okun et al., 2010), suggesting that models like this, based upon a relationship between post-synaptic potentials and field potential, may provide useful insight. It is a construct that simulates the hypothesis of Figures 7A-C, which is a mechanism for what role some (not all) rhythms might play in cortical processing. This heuristic is meant to synthetically generate data of known $1 /$ fbroadband structure with influence of nested oscillation. It is not meant to serve as an accurate physiological model, but rather as a means to validate our signal processing techniques on synthetic data that has approximately similar statistics as empirical data, but with known underlying structure. They are synthesized in the following steps:

Step 1:Action potentials (AP - “spikes") with Poisson-distributed inter-spike intervals arrive from a pre-synaptic cortical pyramidal neuron. We model 6000 of these and assign a random synaptic weight on the interval -1 to 1 to each synapse. The instantaneous AP rate, $\varpi_{m}$, is modulated as a function of task, so that the probability of an AP is higher during the simulated visual search time. This can be formalized as:

$\bar{\varpi}_{m}^{0}(t)=\left\{\begin{array}{l}1 \ni \eta<\rho\left(t_{k}\right) \\ 0 \ni \eta>\rho\left(t_{k}\right)\end{array}\right.$

where $\eta$ is a random variable uniformly distributed on the interval $[0,1]$ and $\rho\left(t_{k}\right)$ is a variable threshold that corresponds to the mean population spike rate within the $k$ th epoch (e.g., $\rho\left(t_{k}\right)=$ "firing rate"/"sampling rate"). The maximum firing rate was set to 40 spikes/s. During simulated ISI epochs, $\rho\left(t_{k}\right)$ was set to $25 \%$ of maximum firing rate, and during each simulated visual search epoch the value of $\rho\left(t_{k}\right)$ was set to a value drawn from a uniform distribution on the range 55-100\% of maximum firing rate. 
Step 2: Each AP produces stereotyped transient post-synaptic current with a sharp rise, and an exponential decay of timescale $\tau_{s}=(2 \pi 75 \mathrm{~Hz})^{-1}=2.1 \mathrm{~ms}$ consistent with empirical measurement (Sabatini and Regehr, 1996). Following Lindén et al. (2010), the timecourse of the synaptic current induced by a single AP is:

$I_{S}(t)=\eta\left(t / \tau_{s}\right) \mathrm{e}^{1-t / \tau_{s}} \theta(t)$, where $\eta$, in this case, is a "synaptic strength" drawn randomly from a uniform distribution on the range $[-1,1], \tau_{s}$ is the time constant of the synapse, and $\theta(t)$ is a step function (i.e., "Heavyside function"). The AP timeseries is convolved (* denotes convolution operation) with the synaptic current shape, $I_{S}$.

$\bar{\omega}_{m}=\bar{\varpi}_{m}^{0} * I_{S}$

Step 3: Synaptic inputs from 6000 synapses, “ $m$," are summed at each point in time:

$W(t)=\sum_{m} \varpi_{m}(t)$

and charge integrates over time, perturbing the trans-membrane difference in charge concentration between the inside and outside of the neuron (Connor and Stevens, 1971).

Step 4: The temporal integration and Ohmic leakage that produce the broadband $B(t)$ are governed by:

$\frac{d B(t)}{d t}=-\alpha B(t)+W(t)$.

The timecourse of $B(t)$ is determined iteratively. Note that, in more comprehensive and subtle simulations, the effect of rhythmic input (through back-propagation or otherwise), might be incorporated into this step.

Step 5: A cortical rhythm $\zeta(t)=r_{\zeta}(t) \cos \left(2 \pi f_{0} t+\Delta\left(t_{k}\right)\right)$, is simulated, with center frequency $f_{0}$ set to $15 \mathrm{~Hz}$. The amplitude $r_{\zeta}(t)$ is set to zero during simulated visual search epochs, and takes on a non-zero value drawn randomly from a uniform non-zero distribution during ISI epochs (determining the strength of the modulation). A different random phase shift, $\Delta\left(t_{k}\right)$, is also added to each epoch to prevent inter-trial coherence in the mean spectrogram.
This rhythm contributes to the simulated membrane potential of a neuron in two ways. Firstly, it multiplicatively modulates the broadband process:

$V_{B B}(t)=(1+\zeta(t)) B(t)$

Secondly, the rhythm contributes directly to the potential:

$V_{\zeta}(t)=A_{\zeta} r_{\zeta}(t) \cos \left(2 \pi f_{0} t+\Delta\left(t_{k}\right)+\phi_{C}\right)$, where $\phi_{c}$ is the coupling phase (which we set to $\pi / 4$ ), and $A_{\zeta}$ is the overall amplitude of the rhythmic contribution, linked to match the variation in the underlying noise: $A_{\zeta}=A_{\zeta}^{0}\left(\sigma_{\zeta(t)} / \sigma_{V_{B B}(F, t)}\right)$ and $\sigma$ denotes the standard deviation over time, and $V_{B B}(F, t)$ is the band-pass filtered $V_{B B}(t)$ for $F=\left\{f_{0}-2 \mathrm{~Hz}\right.$ to $\left.f_{0}+2 \mathrm{~Hz}\right\}$. Then the simulation contribution to the potential from one neuron is $V_{B B}(t)+V_{\zeta}(t)$. In this simulation, we use this quantity to approximate the timecourse that gives rise to our measured potential.

Step 6: Simulation is performed for the summation of 10 such model neurons. The broadband change is a stochastic process, different in each neuron although the timecourse of firing probability, $\rho\left(t_{k}\right)$, is the same at each synapse, and in each neuron. The timing and phase of the rhythm is also fixed across neurons (because it is "synchronized"). The resulting timeseries was analyzed in the same manner as the cortical data were.

The inhibition through synchronization aspect of the model is forced into effect by having the firing rate, $\rho\left(t_{k}\right)$, constructed to be low when $r_{\zeta}(t)$ is non-zero, and $\rho\left(t_{k}\right)$ high as $r_{\zeta}(t) \rightarrow 0$. More sophistocated network simulations should exhibit this as an emergent property.

\section{NEGATIVE CONTROL VALIDATION ON BROWN NOISE (FIGURE 9)}

We tested the methods on pure brown noise that was generated by creating a timeseries with property $P(f)=f^{-2}$ by taking a random white noise variable $\eta$ uniformly distributed on the interval $[-0.25,0.25]$ during simulated ISI, and $[1,1]$ during simulated visual engagement. It was then temporally integrated to give it brown noise structure, and high-passed at $1 \mathrm{~Hz}$ to avoid DC offset issues. Analysis was then performed in an identical manner as with data and the above simulation to demonstrate a negative control (Figure 9). 